\title{
Virtual Notre-Dame: Preliminary results of real-time auralization with choir members
}

\author{
(Invited Paper) \\ Nolan Eley, Sarabeth Mullins, Peter Stitt, and Brian FG Katz \\ Sorbonne Université, CNRS, UMR 7190, Institut Jean Le Rond d'Alembert, Paris, France \\ nolan.eley@sorbonne-universite.fr
}

\begin{abstract}
Heritage acoustics studies are typically constructed around a listener's perspective, using prerecorded source material. The room acoustic response is generated via numerical simulation, such as via geometrical acoustics with proper model calibration. As part of a series of studies investigating the acoustics of heritage spaces and the impact of the acoustics on musical performance, a real-time auralization architecture has been developed and adapted to the requirements of recreating the acoustic conditions within the Cathedral of Notre-Dame de Paris for a choir ensemble. This paper reviews the processing architecture of the system, expanding on previous Experimental Virtual Archaeological Acoustics (EVAA) developments, and an objective level calibration performed for reproduction via headphones and a 32-channel loudspeaker array. A preliminary subjective evaluation was carried out with members of a medieval choir, having experience in Notre-Dame, concerning qualities of the rendering and the calibration procedure. Following the evaluation by this user group, revisions have been made to the rendering architecture with the goal of providing a realtime performance simulator for a four-member ensemble using an anechoic room and individual binaural renderings over open headphones. Further evaluations will concern relevance of additional modifications of the rendering system to improve realism, perception of architectural and acoustic changes related to the historic records of the space, and the impacts of these on musical performances of different genres and periods through objective and subjective analysis of the performances using the real-time auralization rendering system.
\end{abstract}

Index Terms-Heritage acoustics, room acoustics, performance evaluation, real-time auralization

\section{INTRODUCTION}

Room acoustics play an important role in the composition, performance, and perception of music, and the link between acoustics and performance practice has been observed and documented for centuries [1]. Historically, the interaction between acoustics and performance practice has been loosely documented and based on experience, however, the number of serious scientific investigations into the matter has been increasing in recent decades. Seeking to find which acoustic qualities affect musicians and to what extent, these studies have used either real acoustic environments [2]-[4], virtual

Funding has been provided by the European Union's Joint Programming Initiative on Cultural Heritage project PHE (The Past Has Ears, phe.pasthasears.eu), the project PHEND (The Past Has Ears at Notre-Dame, No. ANR-20-CE38-0014, phend.pasthasears.eu), the Chantier Scientifique CNRS/MC Notre-Dame, the CNRS Mission for Transversal and Interdisciplinary Initiatives (MITI), and the Paris Seine Graduate School Humanities, Creation, Heritage, Investissement d'Avenir (No. ANR-17-EURE-0021 Foundation for Cultural Heritage Science) acoustic environments (VAE) [5]-[8], or both [9]-[12]. While the findings have been mixed, some general trends can be extracted. For example, reverberation time tends to have one of the most measurable effects on a musician's performance [5]-[7], [10], [11]. More recently, however, spatially defined acoustic parameters have emerged as showing great importance compared to more traditional omnidirectional parameters [3]. Timbre and loudness tend to be among the most affected performance parameters [4], [6], [8], [11]. Performance parameters related to timing have also been described in many studies as having some correlation with reverberation time, [5]-[7], [10] although the exact correlation varies. One other important conclusion found in several studies is that consistent patterns of adaptation sometimes emerge in individual musicians even if there is an absence of common adaptation patterns among a group of musicians [6], [8], [12].

The utilization of VAEs in these types of experiments allow the subject to experience a wide variety of acoustics (including acoustic spaces which no longer exist) in a very short amount of time, making comparisons between different acoustic environments easier and more reliable by avoiding long time intervals between experiences [13]. However, reliable results from such a study depend on an accurate reproduction of the acoustic properties of the space in question [14]. With recent advances in auralization demonstrating a high degree of plausibility [15], [16], studies using VAEs have become more commonplace.

Expanding on previous Experimental Virtual Archaeological Acoustics (EVAA) developments, this preliminary study sought to assess the perceptual and ecological validity of this auralization architecture, specifically for studying the interaction between musicians and acoustics with an eye towards future studies in acoustic heritage. Four professional singers performed in a VAE and provided feedback through questionnaires. The implementation and calibration of the VAE are described in detail. The results of the questionnaire are discussed followed by recommendations for improving the system for use in future studies.

\section{CONTEXT}

The EVAA system is a technical framework used for ongoing research and documentation of acoustic heritage and historically-informed performance utilizing virtual reality and real-time auralization [17] and is developed in the context of a 
suite of ongoing archaeoacoustic research projects occuring at Sorbonne Université [18]-[21]. Studies utilizing similar VAEs to auralize heritage sites have taken a variety of approaches including both a posteriori and real-time convolution of dry audio signals with relevant room impulse responses (RIRs) [18], [22]-[26].

This study is a part of the "Past Has Ears" (PHE) project, an ongoing research consortium investigating and developing a methodology for archaeoacoustic inquiry and using heritage sites in France, Italy, and the United Kingdom as case studies [27]. The study lays the groundwork for further research into the acoustic heritage of Notre-Dame de Paris, the heritage site at the center of the French component of PHE. The EVAA system delivered a virtual reconstruction of the acoustics of the cathedral based on a geometrical acoustics model which was calibrated to measurements taken prior to the 2019 fire as outlined in [18].

The acoustics of Notre-Dame are the subject of ongoing historical, sociological, and musicological interest as a matter of intangible heritage [27], with particular attention paid to the role it may have played in the development of musical practice during the Middle Ages. The School of Notre-Dame was a musical movement active in the 12th and 13th centuries in Paris and is notable for its innovations in the nascent practice of polyphony [28], [29]. Since the School developed in close temporal proximity to the initial opening of the cathedral, musicologists are interested in researching what influence Notre-Dame may have had on the local composers during the time period. A perceptually validated VAE system is therefore relevant to furthering the interdisciplinary research of the acoustic heritage and impact of Notre-Dame.

\section{OVERVIEW}

\section{A. Study Participants}

The four singers who participated in the study are members of "Rue des Chantres", a medieval ensemble with a particular focus on the musical compositions of the Notre-Dame school of polyphony in the 12th and 13th centuries. As specialists in historically informed performance, they have performed alone or as an ensemble inside Notre-Dame de Paris, the Basilique Cathédrale Notre-Dame d'Amiens, and other venues associated with medieval music. As the aim of this study was to perceptually validate the virtual replication Notre-Dame's acoustics prior to the fire of 2019, the musicians were chosen for their familiarity with the acoustics of the cathedral.

The musicians sang excerpts of both sacred and profane medieval repertoire which they had previously prepared. While no time limit or minimum duration was requested of the musicians, the musicians spent between $10 \mathrm{~min}$ to $15 \mathrm{~min}$ interacting with the VAE in each reproduction condition (multichannel loudspeaker and headphone reproduction). The interactions were musician-led, with each participant free to interact with the VAE as they chose. The participants sang both full excerpts and subsections while standing or moving within the VAE. They also interacted with the VAE by singing plo-

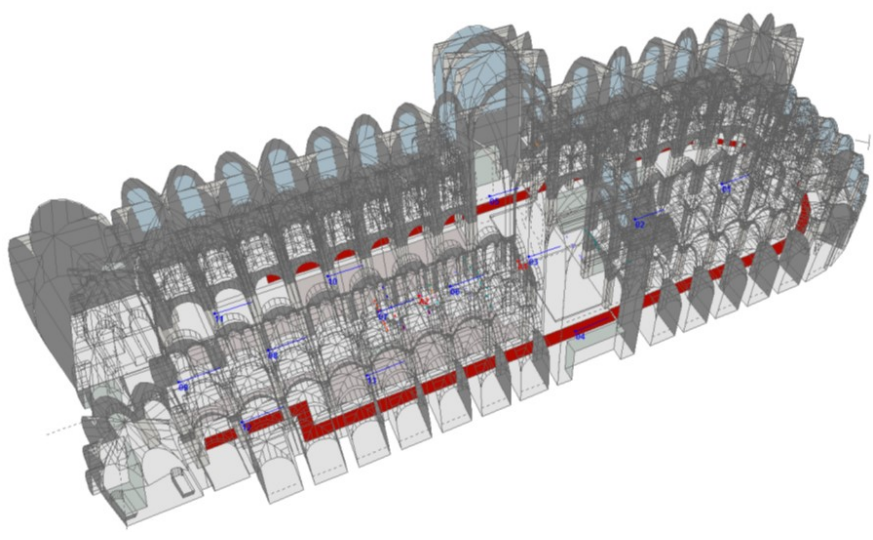

Fig. 1. View of 3D geometrical acoustics model of Notre-Dame.

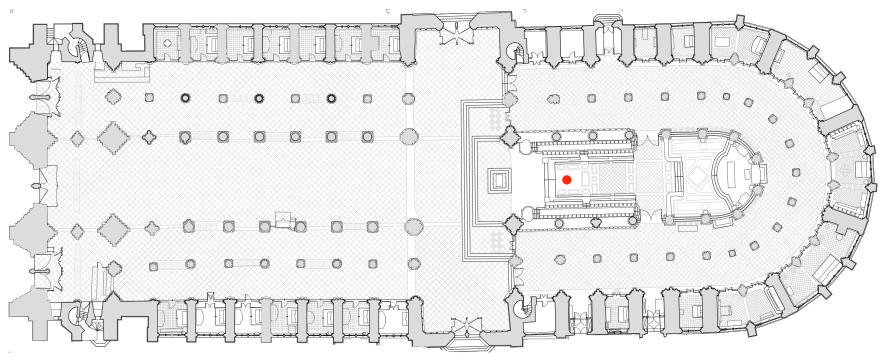

Fig. 2. Floor plan of Notre-Dame with the location of the source/receiver singer-cluster indicated $(\bullet)$.

sives, fricatives, and sibilants independent of a larger musical context.

\section{B. Reproduction System Architecture}

The EVAA system uses a VST3 plugin to perform convolution of a monophonic input with RIRs that allows for dynamic directivity [17]. The previously documented version of the system used a uniform partitioned algorithm with multi-thread processing in two versions: high spatial update latency/minimum convolutions and minimum latency/convolution matrix. The current version used in this study has been updated to use non-uniform partitioned convolution [30], since this allows for more efficient processing and the study used the matrix convolution algorithm in order to ensure good spatial reactivity for the subjects. The directivities were defined as 3rd-order spherical harmonics using a soprano voice directivity pattern [31]. The virtual receiver was 3rdorder Ambisonics. This leads to a $16 \times 16$ filter matrix.

Due to the length of the impulse responses in Notre-Dame, only the first $1 \mathrm{~s}$ was generated via GA simulation, using the CATT-Acoustic (v9.1) and TUCT (v2.0e:1.02) software [32]. The remainder of the RIR was approximated by using the IEM FdnReverb [33], a feedback delay network (FDN) reverb that takes higher order ambisonics (HOA) [34] as input. The TUCT simulation used 2000000 rays while implementing algorithm 1. The source representing the singer location was modelled as a coincident pair with a receiver located roughly $2 \mathrm{~cm}$ away from the source, positioned in the choir of the 


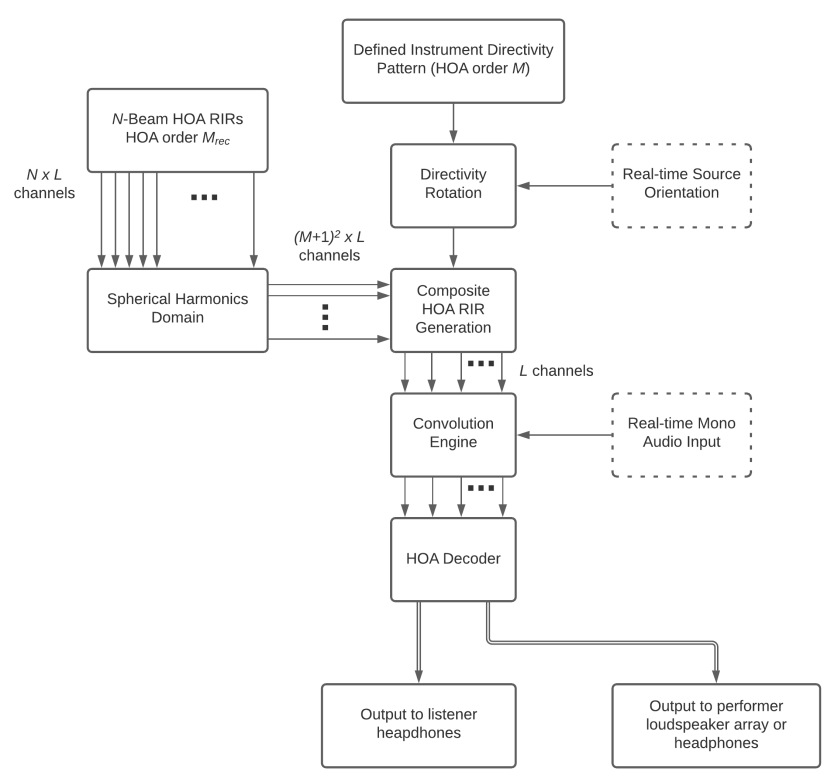

Fig. 3. Schematic diagram of EVAA system architecture.

cathedral. The general location of the singer positions are indicated in red on the floor plan (see Fig. 2), in between the choir stalls oriented along the long axis of the cathedral. The resulting RIRs are preprocessed in order to remove the direct sound as this is not needed for auralization. A more thorough discussion of the creation and calibration of this GA model can be found in [18], [35]. The output of the EVAA plugin was input to the FDN reverb and both outputs were passed to the Ambisonic decoder.

The implementation of the FDN for the remainder of the RIR assumes that the energy decay rate is a single exponential (i.e. linear). This is not truly the case, as was highlighted in [36]. However, for this preliminary study, it is not expected that the secondary decay will contribute significantly to the musicians performance. A supplemental model will include a second FDN to provide this later decay.

The IEM BinauralDecoder [33] was used for binaural rendering when reproducing the EVAA system output via headphone. This plugin uses the MagLS decoding method [37] with the widely used KU 100 dummy head HRTF. Decoding over loudspeakers was done using Spat decoder set to AllRAD [38] and $\max r_{E}$ weighing. A 32-channel loudspeaker array was used for loudspeaker reproduction.

The signal from the singers was captured using a wireless lavalier microphone (DPA 4060) mounted on a headset, with the microphone resting several centimeters from the singer's mouth. The singers also wore a head-mounted motion capture device in order to capture the orientation and movement of the singers for properly rendering the directional characteristics of their voice. An OptiTrack motion capture system and its associated Motive (v1.8.0) software were used for this purpose and the tracking information was routed to Max 8 for further processing.
The experiment took place in the semi-anechoic $\left(T_{30}=\right.$ $0.14 \mathrm{~s}$ at $1 \mathrm{kHz}$ ) motion capture/virtual reality room at Sorbonne Université. The test sessions were run with the plug-in hosted in Max 8, sample rate of $44.1 \mathrm{kHz}$ with buffer sizes of 256 samples $(5.8 \mathrm{~ms})$.

\section{Experimental Overview}

The singers sang their prepared repertoire with the simulated acoustics being conveyed through multichannel loudspeaker reproduction and through headphone reproduction using binaural rendering. In addition to these two performances, subjects were also asked to explore the dynamic directivity of the system by turning while vocalizing in order to assess the impact of its inclusion. As a novel component of the EVAA system architecture, the dynamic directivity module was included to evaluate its perceptual significance to a singer within the VAE. Since the source and receivers are located inside the choir, the early-returning lateral energy from the choir stalls was more likely to be noticeable by the singers as they interacted with the VAE.

The subjects were also each explicitly asked about the overall level of the system response at the beginning of each experience through loudspeakers and headphones in order to assess the effectiveness of the level calibration procedure (see Section IV). The gain was then adjusted to their desired level before beginning. Throughout the experience the subjects were free to comment, and these comments were recorded contemporaneously. The singers also filled out questionnaires related to the perceptual validity of the experience.

In addition to participating as singers, the musicians also participated as listeners, in order to comment on the perceptual validity of a nearby listener position. With an aim at a future expansion of the EVAA system to study ensemble performances, the listener position was modeled after a position that would be typical in an ensemble setup, roughly $1 \mathrm{~m}$ away from the singer. This auralization was created in the same way as the singer position, however the direct sound was not removed. The listeners were set up in another room so they could not hear the singer directly. They were free to comment throughout the process, and they also filled out questionnaires related to the perceptual validity of their experience.

The questionnaires used in this study were aimed at evaluating the validity of the system for music performance. Subjects were asked to rate their experience singing in the simulated acoustics in comparison to their previous experience singing in Notre-Dame in terms of reverberation time, reverberation color, envelopment, and impression of room size. For example, if they rated the reverberation time as longer, they would be indicating that the virtual acoustic environment had a longer reverberation time than Notre-Dame. The questions were administered to the participants as a 5-point Likert scale with the middle point indicating a "similar" rating and the remaining points labeled appropriately for each specific category. The questionnaires were administered to the participants in French.

Additionally, the subjects were asked to rate the virtual acoustics in terms of naturalness and overall acoustic impres- 
sion on a 1 to 5 scale. The same questionnaire was administered to the subjects when they participated as listeners. The questionnaires were based on previous work assessing musicians' evaluations of acoustics [13], [39]-[42]. In addition to the formal questionnaires, the singers' comments were recorded throughout the duration of the experiment.

\section{LeVEl Calibration Procedure}

In preparation for the experiment, a level calibration was performed in order to determine the proper level of the simulated acoustics, relative to the direct sound of the singers. As both headphones and a multichannel speaker array were used to convey the simulated acoustics, a level calibration was performed for each of these methods. Several procedures have been developed for this purpose including [8], [11], [43], [44]. None of these reported methods have achieved perfect subjective validation so this calibration step should be used to achieve a relatively reliable starting level rather than relied upon to determine an absolute fixed level. Minor adjustments can be expected based on musicians' subjective experience, although, these should hopefully not deviate too far as this would indicate a serious inaccuracy in the calibration procedure.

For this study we utilized a slightly modified version of the procedure outlined in [43] which compares the late support parameters, $S T_{\text {late }}$ [45] (see eq. 1), between the VAE and the real acoustic space to produce a gain coefficient by which the simulated acoustics could be amplified or attenuated.

$$
S T_{\text {late }}=10 \log _{10}\left(\frac{\int_{100 \mathrm{~ms}}^{1000 \mathrm{~ms}} h^{2}(t) d t}{\int_{0 \mathrm{~ms}}^{10 \mathrm{~ms}} h^{2}(t) d t}\right)
$$

However, instead of using $S T_{\text {late }}$ measured in the real acoustic space, this parameter was obtained from the GA model as this was the source of the auralization. A set of beam RIRs was produced from the GA model corresponding with the same source and receiver configuration. A loudspeaker (Genelec 8030A) produced an exponential sine sweep which was captured by an omnidirectional microphone (DPA 4060), a few centimeters away, the same microphone and position used for the singers in the VAE. This signal was then run through the EVAA system using the set of RIRs described above using a soprano singer (fortissimo) directivity pattern. This was then captured by an omnidirectional microphone (DPA 4006) positioned $1 \mathrm{~m}$ away. This sweep was deconvolved in order to create an impulse response, which was then used to calculate the $S T_{\text {late }}$ parameter. This measure was compared to the same parameter calculated from the RIR produced by the GA model and a difference, in $\mathrm{dB}$, was calculated between the two. This gain was then applied to the output of the system during the experiments.

The level calibration procedure for the headphones was essentially the same as the above, only in place of the omnidirectional microphone (DPA 4006), a binaural dummy head was used (Neumann KU 80, DPA 4060 microphones placed at the entrance of each artificial ear canal) and a pair of open headphones (Sennheiser HD 600) placed over the ears.

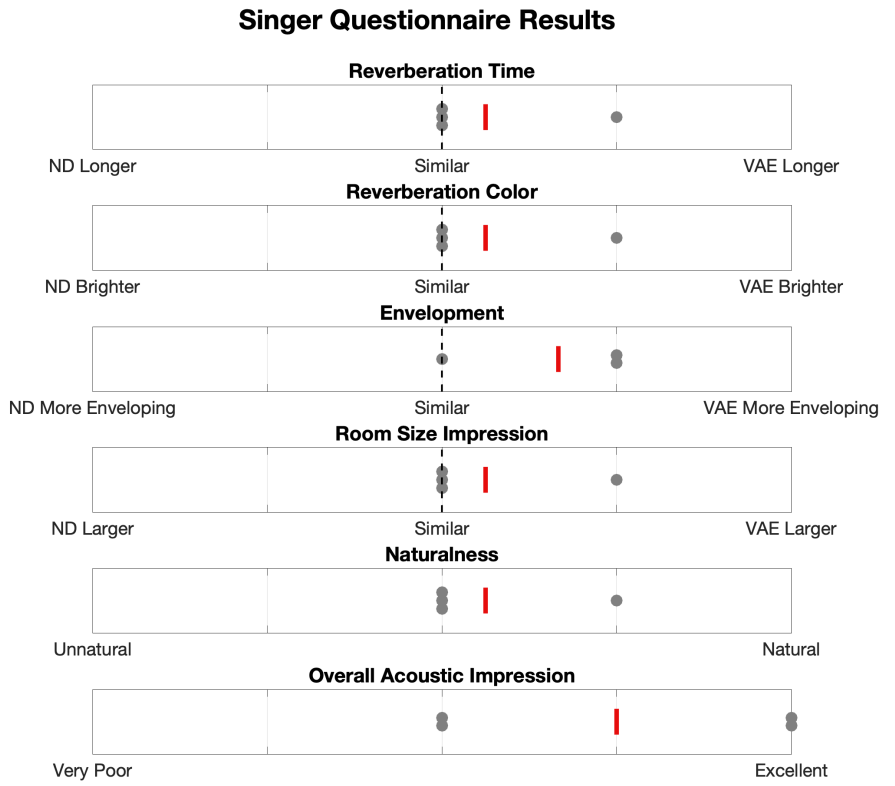

Fig. 4. Individual responses $(\bullet)$ and means $(\mid)$ to singer questionnaire.

Listener Questionnaire Results

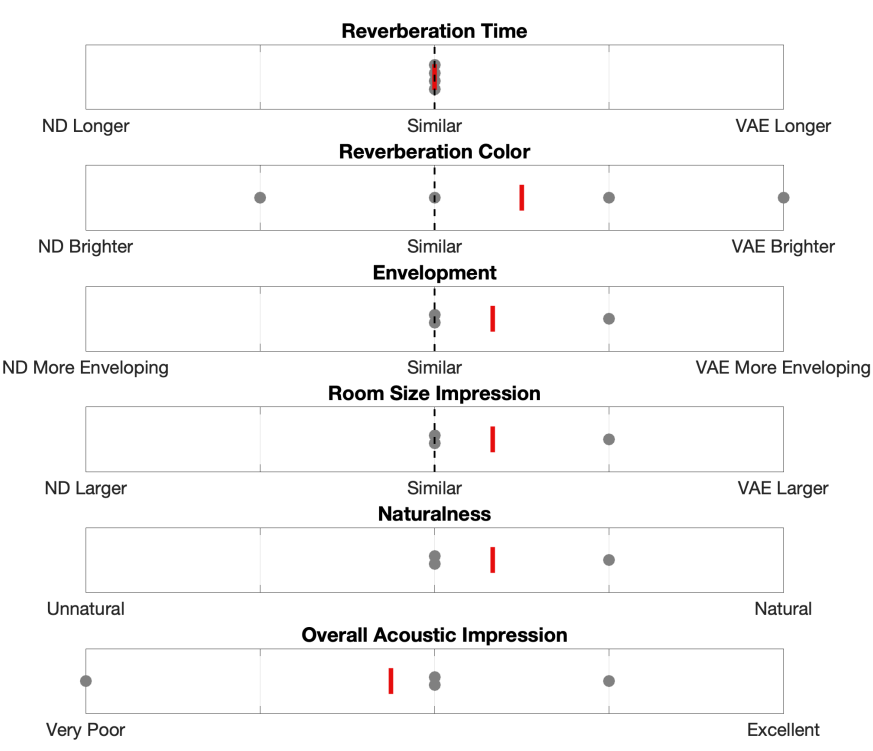

Fig. 5. Individual responses $(\bullet)$ and means $(\mid)$ to listener questionnaire.

\section{Results AND Discussion}

The results of both the listener and singer questionnaires (see Fig. 4 and Fig. 5) indicate that the VAE was evaluated as being relatively similar to Notre-Dame. However, this is according to sample of 4 participants who based their comparisons on previous experiences which occurred at least two years prior to this study. Therefore, any conclusions drawn from this experiment should take this into consideration.

In terms of naturalness, the virtual acoustics were rated, on average, as "somewhat natural" while the mean overall acoustic impression of the singers was a four out of five, 
however, the listener position was judged slightly below three.

In addition to these questionnaires, we also asked each singer to comment on the dynamic directivity of the system. Three out of four singers said that they could not notice a difference in the sound if they rotated while vocalizing. However, one singer commented that he noticed a slight difference when rotating that added to a sense of envelopment. The singers were also asked to compare their experience singing through loudspeaker playback with their experience with playback through a pair of open headphones. Two of the singers preferred the simulated acoustics being conveyed through loudspeakers while one singer preferred the playback through headphones. Another singer had mixed feelings, saying that the acoustic feedback from the headphones sounded more realistic but that it was more difficult to hear himself.

In order to gauge the effectiveness of the level calibration procedure, each of the singers was asked about the overall level of the room response. Through loudspeakers, only one singer requested that the gain of the system be increased by approximately $2 \mathrm{~dB}$. Through headphones, three out of four singers requested a similar gain change. This suggests that the level calibration procedure is generally well-designed, especially for loudspeaker reproduction. However, the disparity between the desired level through loudspeakers compared to headphones is something that merits further investigation.

Despite rating the reverberation color of the VAE as similar to Notre-Dame, in oral feedback following the recording session the participants noted an unnatural and metallic coloration in the higher frequencies of the VAE. This feedback prompted a closer examination of signal flow through the EVAA system and revealed a fault in the calibration of the IEM FdnReverb mentioned in Section III-B. The FDN portion of the system was recalibrated to bring the total synthetic IR of the EVAA system into closer alignment with the measured RIRs of NotreDame. Further examination is required to determine if the FDN settings were responsible for the entirety of the coloration issue.

\section{CONCLUSIONS AND FUTURE WORK}

When presented in isolation, the calibrated EVAA environment was perceived as generally plausible and similar to Notre-Dame. While this assessment is dependent on the recollection of study participants, it is an encouraging sign that development on the VAE is progressing towards realism. The inclusion of dynamic directivity in the system architecture did not appear to tangibly improve the participant's experience of the system. As most participants assumed a singing posture quickly and did not deviate from the posture and position unless prompted, it is thought that future iterations of this system intended for use by choral ensemble may not require motion tracking to maintain immersion. Additionally, the gain initialization and calibration procedure seems to work well for setting preliminary levels prior to participant interaction with the VAE, especially with the multichannel loudspeaker reproduction system.
The study also revealed room for improvement, most especially regarding the high-frequency coloration noted by participants. Participant feedback prompted a re-examination of the FDN used to simulate the late reverberation of the cathedral, and a flaw in the initial calibration was discovered as a result. Further inquiry is needed to determine if the FDN was the sole source of coloration in the reproduction. The musicians also reported a general preference for the multichannel loudspeaker system rather than binaural headphones. Since the eventual goal of the current project is to examine the musicians' performance as an ensemble, future work will focus on addressing musician concerns about comfort, low perceptions of envelopment, and difficulties hearing oneself while singing. Development on the EVAA system architecture will continue in preparation for a larger installation supporting experiments with the full ensemble.

\section{ACKNOWLEDGMENT}

The authors would like to thank the musicologists and musicians who participated in the study. The authors would also like to acknowledge Franck Zagala and David PoirierQuinot for helpful advice and support.

\section{REFERENCES}

[1] K. Schiltz, "Church and chamber: the influence of acoustics on musical composition and performance," Early Music, pp. 64-80, Feb. 2003.

[2] W. Chiang, S.-t. Chen, and C.-t. Huang, "Subjective Assessment of Stage Acoustics for Solo and Chamber Music Performances," Acta Acust united Ac, vol. 89, pp. 848-856, 2003.

[3] L. Panton, M. Yadav, D. Cabrera, and D. Holloway, "Chamber musicians' acoustic impressions of auditorium stages: Relation to spatial distribution of early reflections and other parameters," J Acoust Soc Am, vol. 145, pp. 3715-3726, June 2019, doi:10.1121/1.5111748.

[4] P. Luizard, J. Steffens, and S. Weinzierl, "Singing in different rooms: Common or individual adaptation patterns to the acoustic conditions?," J Acoust Soc Am, vol. 147, pp. EL132-EL137, Feb. 2020, doi: $10.1121 / 10.0000715$.

[5] K. Kato, K. Ueno, and K. Kawai, "Musicians' Adjustment of Performance to Room Acoustics, Part III: Understanding the Variations in Musical Expressions," J Acoust Soc Am, vol. 123, pp. 3610-3610, May 2008, doi:10.1121/1.2934797.

[6] K. Kato, K. Ueno, and K. Kawai, "Effect of Room Acoustics on Musicians' Performance. Part II: Audio Analysis of the Variations in Performed Sound Signals," Acta Acust united Ac, vol. 101, pp. 743759, July 2015, doi:10.3813/AAA.918870.

[7] T. Fischinger, K. Frieler, and J. Louhivuori, "Influence of virtual room acoustics on choir singing.," Psychomusicology: Music, Mind, and Brain, vol. 25, no. 3, pp. 208-218, 2015, doi:10.1037/pmu0000117.

[8] S. Amengual Garí, M. Kob, and T. Lokki, "Analysis of trumpet performance adjustments due to room acoustics," in Proc Intl Symp Room Acoustics, (Amsterdam), Sept. 2019.

[9] A. H. Marshall, D. Gottlob, and H. Alrutz, "Acoustical conditions preferred for ensemble," J Acoust Soc Am, vol. 64, pp. 1437-1442, Nov. 1978, doi:10.1121/1.382121.

[10] K. Kawai, K. Kato, K. Ueno, and T. Sakuma, "Experiment on adjustment of piano performance to room acoustics: Analysis of performance coded into MIDI data.," in Intl Symp Room Acoustics, (Toronto, Canada), June 2013.

[11] Z. Schärer Kalkandjiev, The Influence of Room Acoustics on Solo Music Performances. An Empirical Investigation. PhD Dissertation, Technischen Universität, Berlin, 2015.

[12] P. Luizard, E. Brauer, and S. Weinzierl, "Singing in physical and virtual environments: how performers adapt to room acoustical conditions," in Proc AES conf Immersive and Interactive Audio, (York, UK), Mar. 2019.

[13] A. Gade, "Investigations of musicians' room acoustic conditions in concert halls. part i : Methods and laboratory experiments," Acustica, vol. 69, pp. 193-203, 1989. 
[14] A. C. Gade, "Acoustics for Symphony Orchestras; status after three decades of experimental research," in Proc Intl Symp Room Acoustics, Aug. 2010.

[15] A. Lindau and S. Weinzierl, "Assessing the Plausibility of Virtual Acoustic Environments," Acta Acust united Ac, vol. 98, pp. 804-810, Sept. 2012, doi:10.3813/AAA.918562.

[16] B. N. Postma, H. Demontis, and B. F. Katz, "Subjective evaluation of dynamic voice directivity for auralizations," Acta Acust united Ac, vol. 103, pp. 181-184, 2017, doi:10.3813/AAA.919045.

[17] B. F. Katz, S. Le Conte, and P. Stitt, "EVAA: A platform for experimental virtual archeological-acoustics to study the influence of performance space," in Intl Sym on Room Acoustics (ISRA), (Amsterdam), Sept. 2019, (url)

[18] B. N. J. Postma, D. Poirier-Quinot, J. Meyer, and B. F. G. Katz, "Virtual Reality Performance Auralization in a Calibrated Model of Notre-Dame Cathedral," in EAA EuroRegio2016, (Porto, Portugal), June 2016.

[19] B. F. Katz, B. Postma, D. Thery, D. Poirier-Quinot, and P. Luizard, "Objective and perceptive evaluations of high-resolution room acoustic simulations and auralizations," in Euronoise, (Crete), pp. 2107-2114, May 2018, (url).

[20] B. F. G. Katz, D. Poirier-Quinot, and B. N. J. Postma, "Virtual reconstructions of the Théâtre de l'Athénée for archeoacoustic study," in Intl Cong on Acoustics (ICA), (Aachen), pp. 303-310, Sept. 2019, doi:10.18154/RWTH-CONV-239683.

[21] B. N. Postma and B. F. Katz, "The influence of visual distance on the room-acoustic experience of auralizations," J Acoust Soc Am, vol. 142, no. 5, pp. 3035-3046, 2017, doi:10.1121/1.5009554.

[22] H. Autio, M. Barbagallo, C. Ask, D. Bard Hagberg, E. Lindqvist Sandgren, and K. Strinnholm Lagergren, "Historically Based Room Acoustic Analysis and Auralization of a Church in the 1470s," Applied Sciences, vol. 11, p. 1586, Jan. 2021, doi:10.3390/app11041586.

[23] B. Boren, D. Abraham, R. Naressi, E. Grzyb, B. Lane, and D. Merceruio, "Acoustic simulation of Bach's performing forces in the Thomaskirche," in Proc EAA Spatial Audio Signal Processing symp, pp. 143-148, EAA, 2019, doi:10.25836/SASP.2019.39.

[24] M. Sender, A. Planells, R. Perelló, J. Segura, and A. Giménez, "Virtual acoustic reconstruction of a lost church: application to an Order of Saint Jerome monastery in Alzira, Spain," Journal of Building Performance Simulation, vol. 11, pp. 369-390, May 2018, doi:10.1080/19401493.2017.1340975.

[25] A. Pedrero, A. Díaz-Chyla, C. Díaz, S. Pelzer, and M. Vorländer, "Virtual restoration of the sound of the Hispanic Rite," in Forum Acusticum, (Krakow), p. 5, Sept. 2014

[26] B. N. Postma, S. Dubouilh, and B. F. Katz, "An archeoacoustic study of the history of the Palais du Trocadero (1878-1937)," J Acoust Soc Am, vol. SI: Room Acoustics Modeling and Auralization, pp. 2810-2821, Apr. 2019, doi:10.1121/1.5095882.

[27] B. F. G. Katz, D. Murphy, and A. Farina, "The Past Has Ears (PHE) : XR Explorations of acoustic spaces as Cultural Heritage," in Intl Conf on Augmented Reality, Virtual Reality and Computer Graphics (SALENTO AVR) (L. De Paolis and P. Bourdot, eds.), vol. 12243 of Lecture Notes in Computer Science, (Salento), pp. 91-98, Sept. 2020, doi:10.1007/9783-030-58468-9_7.

[28] R. H. Hoppin, Medieval music: Richard H. Hoppin. The Norton introduction to music history, New York: W. W. Norton, 1st ed., 1978.

[29] C. Wright, Music and Ceremony at Notre-Dame de Paris, 500-1500. Cambridge (MA): Cambridge University Press, 1989.

[30] W. G. Gardner, "Efficient convolution without input-output delay," J Audio Eng Soc, vol. 43, pp. 127-136, 1995.

[31] N. R. Shabtai, G. Behler, M. Vorländer, and S. Weinzierl, "Generation and analysis of an acoustic radiation pattern database for forty-one musical instruments," J Acoust Soc Am, vol. 141, no. 2, pp. 1246-1256, 2017, doi:10.1121/1.4976071.

[32] B.-I. Dalenbäck, “CATT-Acoustic v9.1 with TUCT v2.0,” Feb. 2016.

[33] Institut für Elektronische Musik und Akustik, "Plug-in Descriptions." (url). IEM Plug-in Suite.

[34] J. Daniel, Représentation de champs acoustiques, application à la transmission et à la reproduction de scènes sonores complexes dans un contexte multimédia. PhD thesis, University of Paris 6, 2000.

[35] B. N. J. Postma and B. F. G. Katz, "Creation and calibration method of acoustical models for historic virtual reality auralizations," Virtual Reality, vol. 19, pp. 161-180, Nov. 2015, doi:10.1007/s10055-015-02753.
[36] B. Katz and A. Weber, "An Acoustic Survey of the Cathédrale NotreDame de Paris before and after the Fire of 2019," Acoustics, vol. 2, pp. 791-802, Nov. 2020, doi:10.3390/acoustics2040044.

[37] C. Schörkhuber, M. Zaunschirm, and R. Höldrich, "Binaural Rendering of Ambisonic Signals via Magnitude Least Squares," Fortschritte der Akustik - DAGA 2018, no. March, pp. 339-342, 2018.

[38] F. Zotter and M. Frank, "All-round ambisonic panning and decoding," J Audio Eng Soc, vol. 60, no. 10, pp. 807-820, 2012.

[39] L. A. Miranda Jofre, D. A. Cabrera, M. Yadav, A. Sygulska, and W. L. Martens, "Evaluation of stage acoustics preference for a singer using oral-binaural room impulse responses," J Acoust Soc Am, vol. 133 , pp. 3402-3402, May 2013, doi:10.1121/1.4805922.

[40] T. Lokki, "Sensory evaluation of concert hall acoustics," in Proc Mtgs Acoust, (Montreal, Canada), pp. 032004-032004, 2013, doi:10.1121/1.4800481

[41] A. Giménez, R. M. Cibrián, and S. Cerdá, "Subjective Assessment of Concert Halls: a Common Vocabulary for Music Lovers and Acousticians," Archives of Acoustics, vol. 37, pp. 331-340, Nov. 2012, doi:10.2478/v10168-012-0042-3.

[42] D. Ko, W. Woszczyk, and J. Hong, "Augmented stage support in ensemble performance using virtual acoustics technology," J Acoust Soc Am, vol. 133, pp. 3402-3402, May 2013, doi:10.1121/1.4805923.

[43] I. Laird, P. Chapman, and D. Murphy, "Energy-based calibration of Virtual Performance Systems," in 15th Intl Conf Digital Audio Effects, DAFx 2012 Proc, 2012

[44] J. S. Brereton, Singing in Space(s): Singing performance in real and virtual acoustic environments-Singers' evaluation, performance analysis and listeners' perception. PhD Dissertation, University of York, York, Aug. 2014.

[45] "ISO 3382-1(E) Acoustics - Measurement of room acoustic parameters - Part 1: Performance spaces," standard, International Organization for Standardization, 2009. 